\title{
Novel circRNA_0071196/miRNA-19b-3p/CIT axis is associated with proliferation and migration of bladder cancer
}

\author{
ZAN LIU*, YANG YANG* ${ }^{*}$, ZHE YANG, SHUNYAO XIA, DASEN LIN, BANG XIAO and YOUCHENG XIU \\ Department of Urology, The First Affiliated Hospital of Harbin Medical University, \\ Harbin, Heilongjiang 150001, P.R. China
}

Received October 14, 2019; Accepted June 19, 2020

DOI: $10.3892 /$ ijo.2020.5093

\begin{abstract}
Circular RNAs (circRNAs) are non-coding RNAs that are connected at the $3^{\prime}$ and $5^{\prime}$ ends by an exon or intron. Studies increasingly show that circRNAs play an important role in tumorigenesis by acting as a 'sponge' for microRNAs (miRNAs), which abrogates the latter's effect on their target mRNAs. To identify a possible circRNA/miRNA/mRNA network in bladder cancer (BCa), we analyzed the circRNA and mRNA expression profiles of $\mathrm{BCa}$ and adjacent normal bladder tissues. A total of 127 circRNAs and 1,612 mRNAs were differentially expressed in the tumor tissues, and were primarily associated with cancer-related pathways. A competing endogenous RNAs (ceRNA) network was then constructed which predicted a regulatory axis of circRNA_0071196, miRNA-19b-3p and its target gene citron Rho-interacting serine/threonine kinase (CIT). Luciferase reporter assay validated the relationship between circRNA_0071196 and miRNA-19b-3p and of the latter with CIT. Furthermore, CIT was overexpressed in the BCa tissues, and was found to be correlated with metastasis and tumor histological grade. Knockdown of CIT in the human bladder cancer cell line 5367 significantly inhibited the proliferation, migration and colony formation capacity of the cells, and also upregulated the mediators of the p53 and RhoA-ROCK signaling cascades that regulate cell cycle and migration. Taken together, our findings indicate that circRNA-0071196 upregulates CIT levels in BCa by sponging off miRNA-19b-3p, and the circRNA_0071196/miRNA-19b-3p/CIT axis is a potential therapeutic target in $\mathrm{BCa}$.
\end{abstract}

Correspondence to: Dr Youcheng Xiu, Department of Urology, The First Affiliated Hospital of Harbin Medical University, 23 Youzheng Street, Harbin, Heilongjiang 150001, P.R. China E-mail: zanliu@hotmail.com

*Contributed equally

Key words: circRNA_0071196, CIT, bladder cancer, proliferation, RhoA-ROCK signaling

\section{Introduction}

Bladder cancer $(\mathrm{BCa})$ is the tenth most commonly diagnosed cancer worldwide, with a four-fold higher incidence in men compared to women. Globally, it is the sixth most common cancer in men and ranks ninth as the cause of cancer-related deaths (1). It is rarely diagnosed in individuals younger than 40 years, and occupational exposure to carcinogens is a major risk factor $(2,3)$. Although more common in USA than in Asia, the incidence and mortality rates of $\mathrm{BCa}$ have increased significantly in China in recent years (4). Despite therapeutic interventions such as surgery, chemotherapy and radiotherapy, the 5-year survival of $\mathrm{BCa}$ patients is still low, mainly due to the high recurrence rate $(5,6)$. Therefore, it is essential to identify novel biomarkers of $\mathrm{BCa}$, in order to improve patient prognosis and clinical outcomes.

Non-coding RNAs (ncRNAs) are functional transcripts that are not translated to proteins and modulate gene expression at the transcriptional and post-transcriptional level (7). They are frequently dysregulated in cancer, indicating a prognostic relevance (8). CircRNAs are a class of covalently closed endogenous ncRNAs (9) that are differentially expressed in various types of cancers, such as liver carcinoma, stomach carcinoma and colorectal carcinoma, and correlated with tumor development and progression $(10,11)$. Although the exact function of circRNAs is poorly understood, there is evidence indicating that they act as 'sponges' of the microRNAs (miRNAs) through competitive binding and abrogate the effect of the latter on mRNA translation (12). In addition, circRNAs with the same microRNA response elements (MREs) act as competing endogenous RNAs (ceRNAs) and form a regulatory network. Recent studies have unearthed several ceRNA networks that are dysregulated during cancer (12-14). However, the exact number of circRNAs that act as miRNA sponges is still unknown (15). MicroRNAs are a category of endogenous short non-coding RNAs that can bind to the 3'-untranslated region (3'-UTR) of mRNAs and inhibit their translation (16). miRNA dysregulation has been implicated in the initiation and progression of various cancer types through multiple mechanisms $(17,18)$. In the present study, we discovered a novel ceRNA network comprising circRNA_0071196, CIT and miRNA-19b-3p in $\mathrm{BCa}$ tissues. Based on molecular and bioinformatics analyses, we surmised that circRNA_0071196 upregulates CIT in BCa cells by competitively binding to miRNA-19b-3p. Furthermore, 
knockdown of CIT inhibited the proliferation and migration of $\mathrm{BCa}$ cells in vitro. Taken together, this novel regulatory axis is essential for $\mathrm{BCa}$ progression, and is a potential diagnostic marker and therapeutic target.

\section{Materials and methods}

Patients and samples. Bladder carcinoma $(\mathrm{n}=80)$ and para-carcinoma tissues $(n=30)$ were resected from $\mathrm{BCa}$ patients who underwent surgery at the Department of Urology, The First Affiliated Hospital of Harbin Medical University, China from September 2017 to September 2018. The mean age of the patients was 63.63 years (age range, 41-80) and the sex distribution was 25 females and 55 males. The tumor tissues were obtained by transurethral resection and the para-carcinoma tissues were acquired at a distance of $>2.0 \mathrm{~cm}$ from the tumor margin, and cut into $0.8-1.2 \mathrm{~cm}$ pieces. All tissues were snap-frozen in liquid nitrogen and independently identified by two pathologists. The study was approved by the Ethics Committee of The First Affiliated Hospital of Harbin Medical University, and written informed consent was obtained from all patients.

$R T-q P C R$. Total RNA was extracted from 20 pairs of BCa samples and the transduced 5637 cells, and reverse transcribed to cDNA using the SuperScript ${ }^{\mathrm{TM}}$ III Reverse Transcriptase kit (Invitrogen/Thermo Fisher Scientific, Inc.) according to the manufacturer's instructions. The thermocycling conditions of RT-qPCR were $95^{\circ} \mathrm{C}$ for $10 \mathrm{~min}$, followed by 40 cycles at $95^{\circ} \mathrm{C}$ for $30 \mathrm{sec}$, and $60^{\circ} \mathrm{C}$ for $60 \mathrm{sec}$. The relative expression levels of the relevant transcripts were analyzed by RT-qPCR, and the primer sequences are listed in Table I. GAPDH was used as the internal control for circRNAs and mRNAs, and U6 for miRNAs. The $2^{-\Delta \Delta \mathrm{Cq}}(19)$ method was used to quantify these transcripts, and assays were performed in triplicates.

Microarray analysis. Four pairs of carcinoma and para-carcinoma tissues were collected from BCa patients at our hospital in 2017 for microarray analysis. For circRNA hybridization, the latter were first enriched by degrading the linear RNAs with Rnase R, amplified with random primers, and transcribed into fluorescent cRNA (Arraystar Super RNA Labeling Kit; Arraystar). The labeled cRNAs were hybridized onto the Arraystar Human circRNA Array V2 (8x15K, Arraystar), and the arrays were scanned using the Agilent Scanner G2505C. The significant differentially expressed genes (DEGs) and circRNAs (DEcircRNAs) were identified based on fold-change $\geq 1.5$ and $\mathrm{P}<0.05$.

RNA sequencing array. Total RNA was extracted from four pairs of frozen carcinoma and para-carcinoma tissues using TRIzol (Invitrogen/Thermo Fisher Scientific, Inc.) according to the manufacturer's instructions. After quantitative analysis and quality inspection, RNA-Seq libraries were synthesized using the KAPA Stranded RNA-Seq Library Prep Kit (Illumina, USA) as previously described (20). which included RNA fragmentation, random hexamer-primed first-strand cDNA synthesis, dUTP-based second-strand cDNA synthesis, end-repairing, A-tailing, adaptor ligation and library PCR amplification. The libraries were assessed with an Agilent 2100
Bioanalyzer (Illumina, USA), quantified absolutely by qPCR, and sequenced using an Illumina HiSeq 4000 Sequencing System for 150 cycles.

The raw data was uploaded to Gene Expression Omnibus (GEO) (GSE147985, https://www.ncbi.nlm.nih. gov/geo/query/acc.cgi?acc=GSE147985).

$G O$ and KEGG pathway analyses. Gene Ontology (GO) analysis (http://www.geneontology.org) was performed on the DEcircRNAs and DEGs, and the latter were functionally annotated on the basis of biological processes (BP), cellular components (CC) and molecular functions (MF). GO terms with P-values $<0.05$ were considered as enriched. The Kyoto Encyclopedia of Genes and Genomes (KEGG) pathway analysis (http://www.genome. jp/kegg/) was preformed to identify the significant pathways related to the relevant genes, using P-value $\leq 0.05$ as the threshold.

ceRNA network analysis. Following validation of the DEGs and DEcircRNAs with real time qPCR, those with the same MREs were selected for constructing the circRNA/miRNA/mRNA network. The circRNA-miRNA interactions were predicted by biological algorithms of miRanda (http://www.microrna.org/microrna/home.do) and Targetscan (http://www.targetscan.org/). The miRNA-mRNA interactions were predicted by miRDB (http://www.mirdb. org/). The circRNA/miRNA/mRNA network was then mapped using Cytoscape v 2.8.3 (21).

Cell lines and lentiviral infection. The human bladder cancer cell line 5637 was obtained from the Cell Resource Center, Shanghai Institute for Biological Sciences at the Chinese Academy of Sciences, and maintained at $37^{\circ} \mathrm{C}$ under $5 \% \mathrm{CO} 2$ in RPMI-1640 medium supplemented with $10 \%$ fetal bovine serum (FBS), $100 \mathrm{U} / \mathrm{ml}$ penicillin and $100 \mathrm{U} / \mathrm{ml}$ streptomycin. The lentiviral CIT and control shRNA vectors were designed and synthesized by Shanghai GeneChem Co., Ltd. (Shanghai, China), and the sequences were as follows: CIT, 5'-GCGTC CTCATACCAGGATAAA-3' and control, 5'-TTCTCCGA ACGTGTCACGT-3'. The lentiviral circRNA-0071196 and control shRNA vectors were also designed and synthesized by Shanghai GeneChem Co. Ltd. (Shanghai, China), and the sequences were: circRNA-0071196, 5'-AAGGAACAAGC AGTAGATCAT-3' and control, 5'-TTCTCCGAACGTGT CACGT-3'. The miR-19b-3p inhibitor was synthesized by GenePharma Co. (GenePharma, Shanghai, China). The plasmids were transfected into $293 \mathrm{~T}$ cells using Lipofectamine ${ }^{\circledR} 2000$ (Invitrogen/Thermo Fisher Scientific, Inc.), and the viral supernatants were collected after $48 \mathrm{~h}$, centrifuged, and filtered through a $0.45-\mu \mathrm{m}$ polyvinylidene fluoride membrane. The 5673 cells were incubated with $50 \%$ diluted viral supernatant in complete medium for $48 \mathrm{~h}$, and then selected with $2 \mathrm{ng} / \mathrm{ml}$ puromycin (Thermo Fisher Scientific, Inc.) in fresh medium. Transduction efficiency was monitored under a fluorescence microscope.

Luciferase reporter assay. The psiCHECK2-circRNA0071196-WT, (Promega Corp.) and psiCHECK2-circRNA0071196-Mut luciferase reporter plasmids were constructed by respectively cloning the wild-type (WT) and miR-19b-3p 
Table I. RT-qPCR primer sequences.

\begin{tabular}{lll}
\hline Primer name & \multicolumn{1}{c}{ Primer FW (5'-3') } & \multicolumn{1}{c}{ Primer RV (5'-3') } \\
\hline hsa_circRNA_103758 & GTGGCCGAGGACTTTGATTG & CCTGTAACAACGCATCTCATATT3 \\
hsa_circRNA_404289 & CCTTTTTCTTCTTTCTTTCTGG & TGGAGACTTTACTCTTACCCGT \\
hsa_circRNA_000758 & GGTATTAGGGACACTGGTGG & CTTCTGGCCTTTTGGTTACT3 \\
hsa_circRNA_0006473 & TCAGGTACTCCCGTCGC & CGTTACTCCACCTGGACC \\
miR-19b-3p & GTGCAGGGTCCGAGGT & TGTGCAAATCCATGCAAAACTGA \\
CIT & CAGGCAAGATTGAGAACG & GCACGATTGAGACAGGGA \\
CDK1 & TGGATCTGAAGAAATACTTGGATTCTA & CAATCCCCTGTAGGATTTGG \\
CCND1 & CTAAGATGAAGGAGACCATCCC & AAGGTCTGCGCGTGTTTGCGGAT \\
CCNB1 & TCCAGTTATGCAGCACCTGGCTA & TGCCACAGCCTTGGCTAAATCTT \\
CCNB2 & TGGAAAAGTTGGCTCCAAAG & CTTCCTTCATGGAGAGACATCCTC \\
P53 & TGCTCTTTTCACCCATCTAC & ATACGGCCAGGCATTGAAGT \\
MLC2 & ACCATTCTCAACGCATTCAA & CATCTGGTCAACCTCCTCCT3 \\
MDM2 & ACCTCACAGATTCCAGCTTCG & TTTCATAGTATAAGTGTCTTTTT \\
ROCK1 & AACATGCTGCTGGATAAATCTGG & TGTATCACATCGTACCATGCCT \\
U6 & CTCGCTTCGGCAGCACA & AACGCTTCACGAATTTGCGT \\
GAPDH & TGACTTCAACAGCGACACCCA & CACCCTGTTGCTGTAGCCAAA
\end{tabular}

FW, forward; RV, reverse.

binding site-mutated (MUT) circRNA-0071196 sequence. The psiCHECK2-circRNA-404289-WT, and psiCHECK2circRNA-404289-Mut luciferase reporter plasmids were constructed by respectively cloning the wild-type (WT) and hsa-miR-370-3p binding site-mutated (MUT) circRNA-404289 sequence. The psiCHECK2-circRNA-000758-WT, and psiCHECK2-circRNA-000758-Mut luciferase reporter plasmids were constructed by respectively cloning the wild-type (WT) and hsa-miR-6808-3p binding site-mutated (MUT) circRNA-000758 sequence. The psiCHECK2-circRNA-006473-WT, and psiCHECK2circRNA-006473-Mut luciferase reporter plasmids were constructed by respectively cloning the wild-type (WT) and hsa-miR-6808-3p binding site-mutated (MUT) circRNA006473 sequence. The 5637 cells were seeded into 24 -well plates and cultured for $24 \mathrm{~h}$, and co-transfected with the WT/MUT of the above plasmids and miRNA mimics or the negative control using Lipofectamine 2000 (Thermo Fisher Scientific, Inc.). After culturing for $48 \mathrm{~h}$, firefly and Renilla luciferase activities were quantified using dual luciferase reporter assays (Promega Corp.) according to the manufacturer's instructions.

Immunohistochemistry (IHC). IHC was performed on 4 pairs of $\mathrm{BCa}$ and normal bladder tissues using the SABC kit (Suolaibao) according to the manufacturer's instructions. The sections were incubated with the primary anti-CIT antibody (dilution 1:100; cat. no. ab110897, Abcam) at $37^{\circ} \mathrm{C}$ for $1 \mathrm{~h}$, followed by sequential incubation with biotinylated secondary antibody (dilution 1:1,000; cat. no. ab6721, Abcam) and streptavidin peroxidase for $20 \mathrm{~min}$ each at room temperature. After rinsing the slides, the sections were developed using diaminobenzidine and counterstained with hematoxylin. The stained sections were observed under a light microscope (Olympus
Corp., Japan) at x200 magnification. The staining intensity was graded as follows: 0 , negative; 1 , low; and 2 , high. The percentage of stained cells was scored as: none stained, 0 ; $1-33 \%, 1 ; 34-66 \%, 2$; and $\geq 67 \%, 3$. Both scored values were multiplied to obtained the total CIT score, and samples with CIT $\leq 1$ were considered negative and those with CIT $\geq 3$ as positive.

Western blot analysis. Total proteins were extracted from the transduced 5637 cells, and western blot analysis was performed as previously described (22). The primary antibodies included anti-CIT (dilution 1:5,000) (cat. no. ab86782, Abcam), anti-MLC2 (dilution 1:2,000) (cat. no. ab92721, Abcam), anti-P53 (dilution 1:500) (cat. no. AF6073, Affinity Biosciences), anti-ERK1/2 (dilution 1:1,000) (cat. no. BF8004, Affinity Biosciences), anti-p-ERK1/2 (dilution 1:1,000) (cat. no. AF1015, Affinity Biosciences), anti-CDK1 (dilution 1:1,000) (cat. no. DF6024, Affinity Biosciences), anti-MDM2 (dilution 1:1,000) (cat. no. AF6376, Affinity Biosciences), anti-CCND1 (dilution 1:1,000) (cat. no. AF0931, Affinity Biosciences), anti-ROCK1 (dilution 1:1,000) (cat. no. AF7016, Affinity Biosciences) and anti- $\beta$-actin (dilution 1:15,000) (cat. no. AF7018; Affinity Biosciences). Goat anti-rabbit IgG (dilution 1:2,000) (cat. no. BA1054; Wuhan Boster Biological Technology) was used as the secondary antibody, and the ECL-Plus kit was used to detect protein bands (Amersham Biosciences, USA) as described previously (23). Imaging J V1.53b (National Institute of Health, Bethesda, MD, USA) was used to quantitate the protein.

In vitro assays. Proliferation of the transduced 5637 cells was analyzed using the Cell Counting Kit-8 (CCK-8; cat. no. AR1160-500; Wuhan Boster Biological Technology, Ltd.) according to the manufacturer's instructions. Briefly, the cells 
A

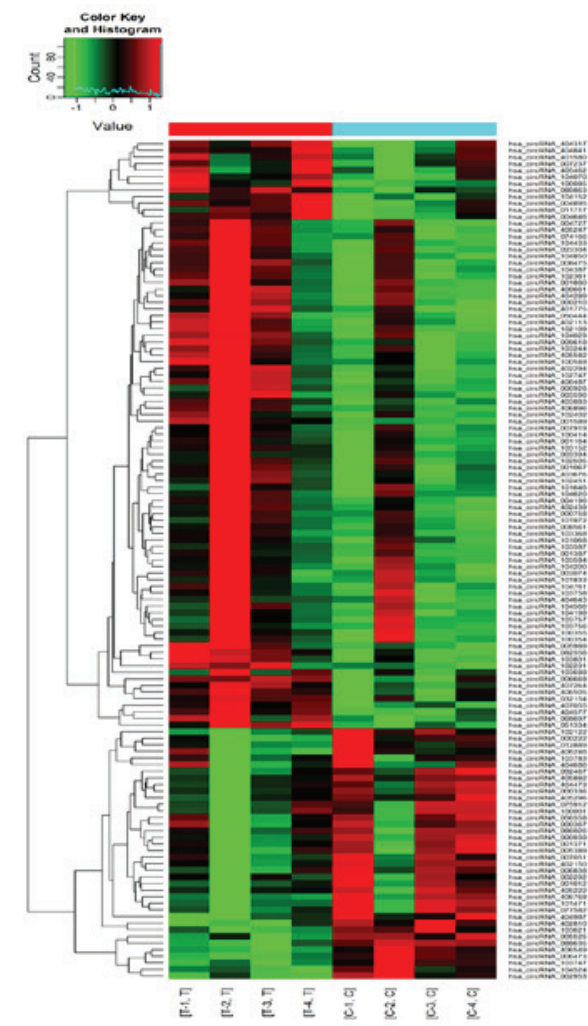

B

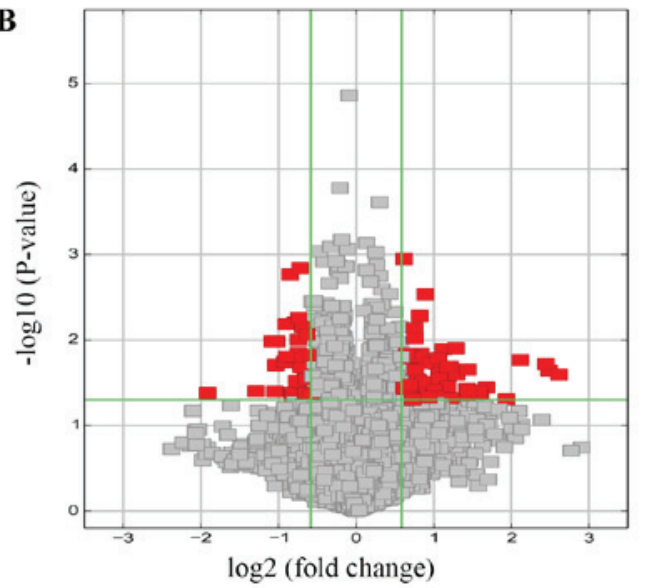

Figure 1. Differentially expressed circRNAs in BCa tissues (T) and adjacent normal tissues (C). (A) The heatmap of DEcircRNAs. (B) Volcano plot showing distribution of circRNAs. The red rectangles represent those with fold-change $>1.5$ and P-value $<0.05$. circRNAs, circulating RNAs; $\mathrm{BCa}$, bladder cancer; DEcircRNAs, differentially expressed cirRNAs.

were seeded in 96 -well plates at the density of $1.5 \times 10^{3}$ cells/well, and cultured for $0,24,48,72$ and $96 \mathrm{~h}$. After adding $10 \mu \mathrm{l}$ CCK-8 solution per well, the cells were incubated in the dark for $1 \mathrm{~h}$, and the absorbance was measured at $450 \mathrm{~nm}$ using Biotek Elx800 spectrometer. The experiment was performed in triplicates. For the migration assay, the cells were seeded onto the upper wells of Transwell chambers (Corning Inc.) at the density of $1 \times 10^{4}$ cells/well in serum-free 1640 , and the lower wells were filled with complete 1640 medium. After a $24 \mathrm{~h}$ incubation at $37^{\circ} \mathrm{C}$, the migrated cells were stained with crystal violet for $30 \mathrm{~min}$ at room temperature, and counted under an inverted microscope at x50 magnification in at least three random fields. For the colony formation assay, the transduced cells were seeded in a 24-well plate at the density of
$2 \times 10^{2}$ cells/well in complete 1640 medium, and cultured for 12 days. The cells were then fixed and stained with $1 \%$ crystal violet at room temperature for $30 \mathrm{~min}$, and the colonies were photographed and counted using a light microscope (magnification, $\mathrm{x} 50$ ).

Statistical analysis. Categorical variables were compared by Fisher exact test, and continuous variables by the Student's t-test. Data are expressed as the means \pm standard deviation. Multigroup comparisons of the means were carried out by one-way analysis of variance (ANOVA) test with post hoc contrasts by Student-Newman-Keuls test. All statistical analyses were performed using SPSS 17.0 (SPSS, Inc.). P $<0.05$ was considered statistically significant. All experiments were repeated in triplicates

\section{Results}

DEcircRNAs in the BCa tissues are associated with cancer-related functions. The expression profiles of 4 pairs of $\mathrm{BCa}$ and normal bladder tissues were analyzed by high throughput microarray, and 127 DEcircRNAs were detected of which 89 were upregulated and 38 were downregulated in the tumor samples (Figs. 1A and B and S1 A and B). Based on their relation with protein-coding genes, the DEcircRNAs were classified as exonic (85.83\%), intronic (8.66\%), sense overlapping $(4.72 \%)$ and antisense $(0.79 \%)$ (Fig. S1C). Furthermore, the DEcircRNAs were distributed across all chromosomes except the Y chromosome (Fig. S1D). Thus, the circRNA profiles of $\mathrm{BCa}$ and normal bladder tissues were distinct. The biological relevance of these DEcircRNAs was determined by the GO and KEGG analyses. The top 10 enriched GO terms (all domains) of the abnormally expressed circRNAs were 'Transcription regulatory region DNA binding', 'Regulatory region nucleic acid binding', 'Transcription regulatory region sequence-specific DNA binding', 'Intracellular part', 'Anatomical structure development', 'Protein binding', 'Intracellular', and 'Positive regulation of cellular process' (Fig. 2A and B). KEGG pathway analysis revealed that the top 10 pathways associated with upregulated (Fig. 2C) and downregulated (Fig. 2D) circRNAs included 'Hepatocellular carcinoma', 'FoxO signaling pathway', 'Proteoglycans in cancer, 'Th17 cell differentiation', 'Th1 and Th2 cell differentiation' and 'MAPK signaling pathway'.

$B C a$ and normal bladder tissues have distinct mRNA profiles. In addition to the circRNAs, the $\mathrm{BCa}$ and adjacent normal tissues also differed in terms of the mRNA expression profile. A total of 1,612 DEGs were identified, including 797 upregulated and 815 downregulated mRNAs (Figs. 3 and S2). The top 10 enriched GO terms in the upregulated and downregulated mRNAs are respectively shown in Fig. 4A and B. The most significantly enriched $\mathrm{BP}, \mathrm{CC}$ and $\mathrm{MF}$ terms among the upregulated mRNAs were 'Chromosome segregation', 'Chromosomal region' and 'Catalytic activity on DNA', respectively, and those among the downregulated mRNAs were 'Muscle system process', 'Contractile fiber part' and 'Actin binding'. KEGG pathway analysis showed that the top 10 pathways associated with the upregulated mRNAs included 'Cell cycle', 'DNA replication' and 'p53 signaling pathway' (Fig. 4C), and those associated with 
A
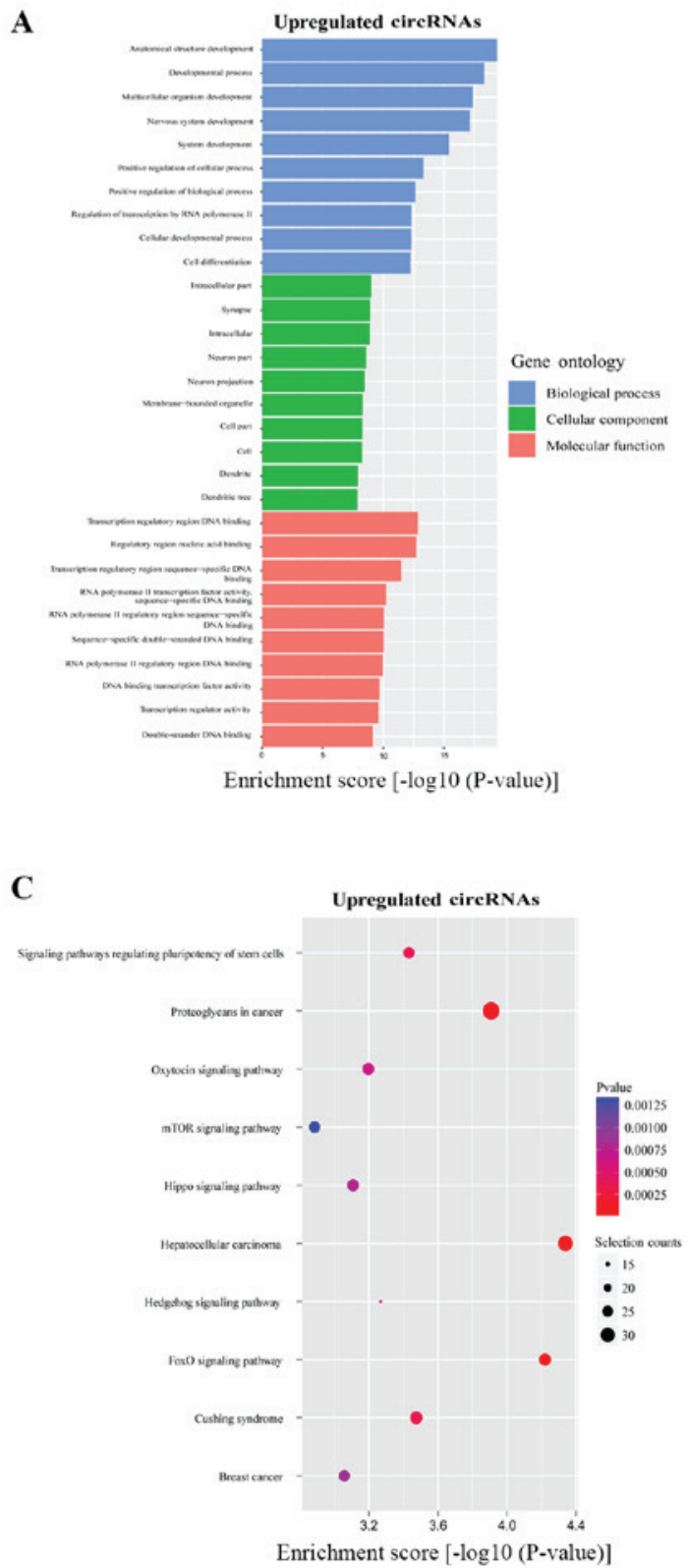

B

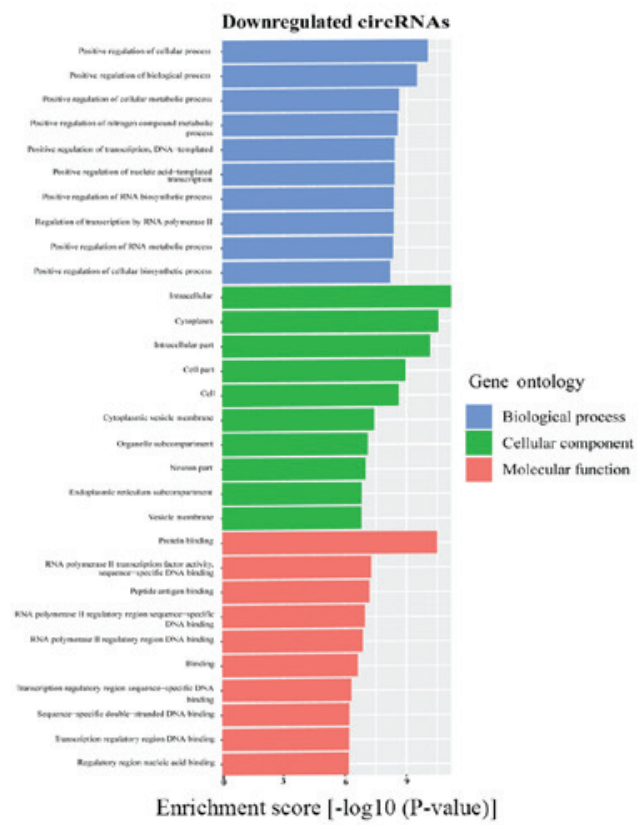

D

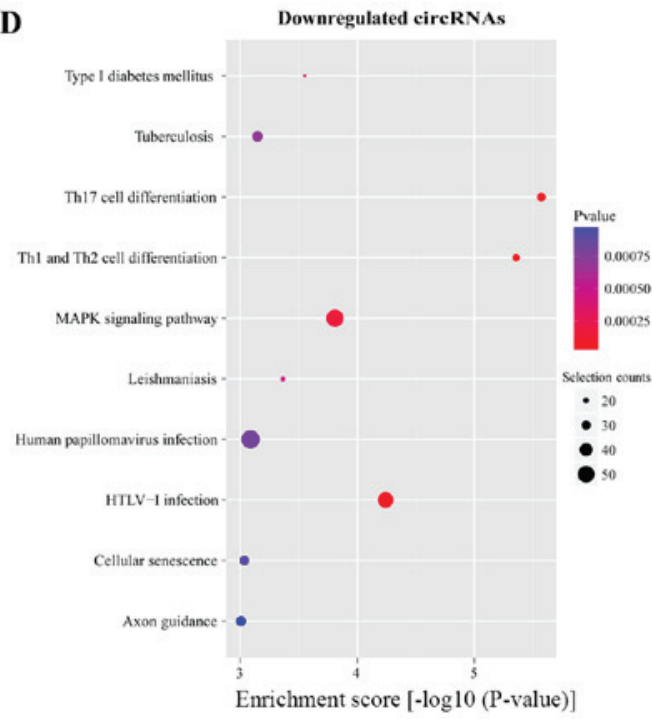

Figure 2. GO and KEGG pathway analyses of the genes encoding DEcircRNAs. (A and B) The top 10 enriched GO terms of the upregulated and downregulated circRNAs. (C and D) The top 10 enriched KEGG pathways of the upregulated and downregulated circRNAs. GO, Gene Ontology; KEGG, Kyoto Encyclopedia of Genes and Genomes; circRNAs, circulating RNAs; DEcircRNAs, differentially expressed cirRNAs.

downregulated mRNAs were mainly in the 'Calcium signaling pathway', 'cGMP-PKG signaling pathway' and 'Vascular smooth muscle contraction' (Fig. 4D). Four circRNAs and 5 mRNAs were selected for verifying the microarray results by RT-PCR, which showed that circRNA_0071196, circRNA_404289, circRNA_000758, cyclin dependent kinase 1 (CDK1), cyclin D1 (CCND1), cyclin B1 (CCNB1), cyclin B2 (CCNB2) and citron Rho-interacting serine/threonine kinase (CIT) were upregulated (Fig. 5A and B), while circRNA_006473 was downregulated (Fig. 5A). These results were consistent with the microarray data (Fig. 5C and D). Compared with other circRNAs, only circRNA_0071196 was positive in the luciferase assay, thus we chose circRNA_0071196 to continue the following study.

circRNA_0071196 and CIT are targets of miR-19b-3p. The ceRNA network was constructed using the DEcircRNAs and DEGs that shared binding sites for MREs, which revealed that circRNA_0071196 and CIT were the interacting partners of miR-19b-3p (Fig. 6). We further predicted the circRNA_0071196/CIT and miR-19b-3p/CIT interactions using the Starbase (starbase.sysu.edu.cn/index.php) and miRanda (http://www.microrna.org/microrna /home. do) programs, respectively. As shown in Fig. 7A, the bases in the seed region of miR-19b-3p were complementary to the circRNA_0071196 and CIT sequences, indicating that circRNA_0071196 likely acts as a sponge for miR-19b-3p in BCa cells, and that CIT is a downstream target of miR-19b-3p. To validate our hypothesis, we analyzed the expression levels of miR-19b-3p in BCa and adjacent normal tissues, and found that the expression of miR-19b-3p exhibited no significant difference in the tumor and para-carcinoma tissues (Fig. 7B). Furthermore, circRNA_0071196 WT 
A

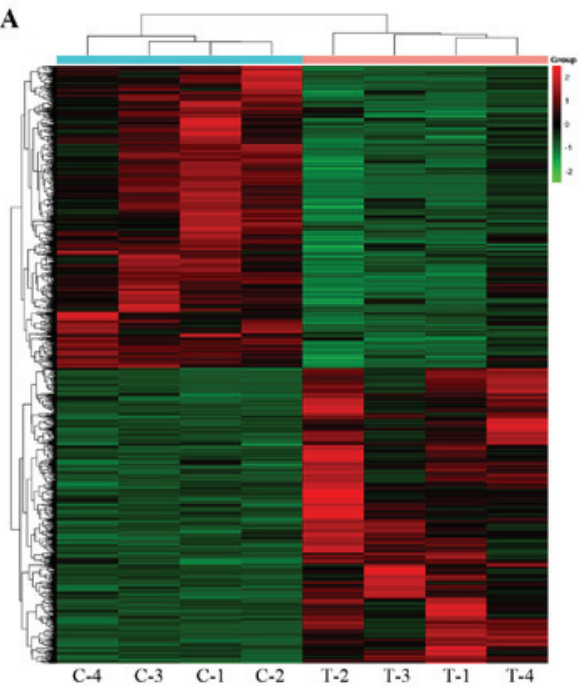

B

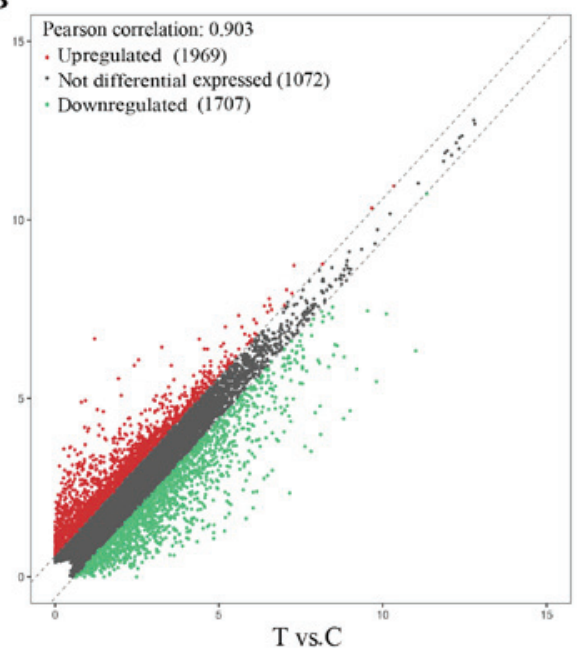

Figure 3. Differential mRNA expression between BCa tissue (T) and adjacent normal tissues (C). (A) Hierarchical clustering of the DEGs in BCa and normal bladder tissues. Red and green color represent the upregulated and downregulated genes, respectively. (B) Scatter plot showing the distribution of the mRNAs, with $\mathrm{x}$ - and $\mathrm{y}$-axes showing the average normalized values of each group ( $\log 2$ scales). The mRNAs above the top black dotted line and below the bottom black dotted line have greater than 1.5 -fold-change, and are respectively upregulated and downregulated. DEGs, differentially expressed genes; BCa, bladder cancer.

A

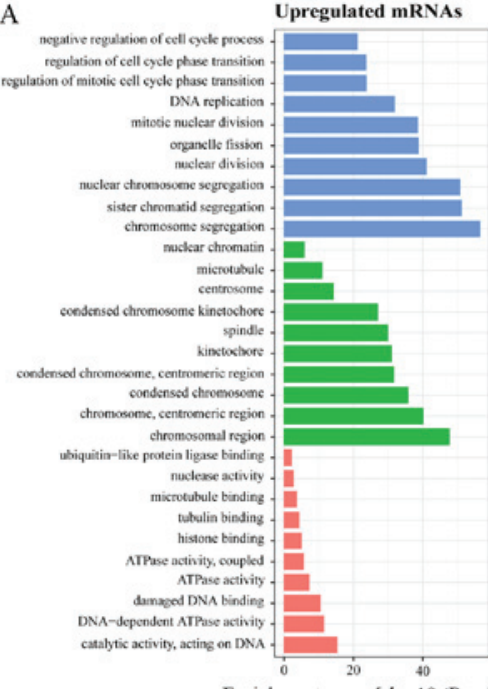

Enrichment score [-log 10 (P-value)]

C

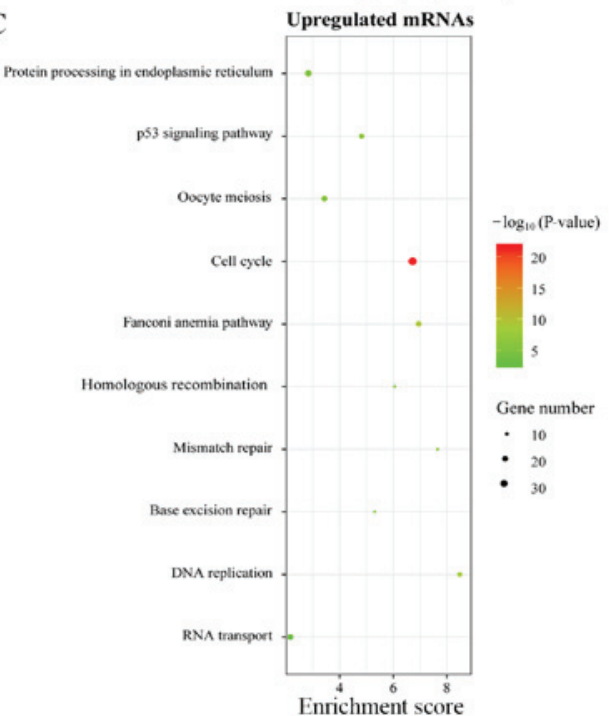



D

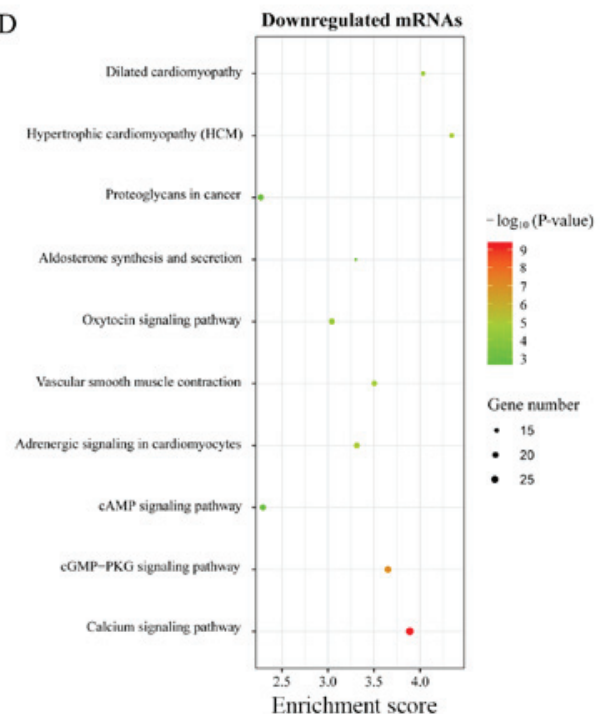

Figure 4. GO and pathway analysis of the DEGs. (A and B) The top 10 enriched GO terms of the upregulated and downregulated mRNAs. (C and D) The top 10 enriched KEGG pathways of the upregulated and downregulated mRNAs. GO, Gene Ontology; DEGs, differentially expressed genes; KEGG, Kyoto Encyclopedia of Genes and Genomes. 



Figure 5. Validation of the microarray analysis. (A and B) Four circRNAs and five mRNAs were randomly selected and tested by RT-qPCR. * $<0.05$ vs. adjacent normal tissues. (C and D) Comparison of qPCR and microarray results. The height of the column represents fold-change (log2 transformation). circRNAs, circulating RNAs; CDK1, cyclin-dependent kinase 1; CCND1, cyclin D1; CCNB1, cyclin B1; CCNB2, cyclin B2; CIT, citron Rho-interacting serine/threonine kinase.

3'-UTR co-transfected with miR-19b-3p showed lower luciferase activity compared to transfection with miR-control. In contrast, no significant difference was seen in the luciferase activity of circRNA_0071196 MT 3'-UTR in the presence of miR-19b-3p mimics or miR-control (Fig. 7C). Similarly, CIT WT 3'-UTR showed distinctly weaker luciferase signals when co-transfected with miR-19b-3p compared to miR-control, whereas that of CIT WT 3'-UTR was unaffected by the miRNA (Fig. 7D). Taken together, circRNA_0071196 acts as a sponge for miR-19b-3p and CIT is the target of miR-19b-3p in BCa cells. Because the luciferase results of other circRNAs in Fig. 5 were negative (data not shown), circRNA-0071196 was selected for subsequent experiments.

circRNA_0071196 and miR-19b-3p are correlated with BCa progression. To further elucidate the biological functions of circRNA-0071196 and miR-19b-3p in BCa, we transfected 5637 cells with si-circRNA-0071196 and miR-19b-3p inhibitor/NC inhibitor. qRT-PCR showed that circRNA-0071196 expression was significantly decreased compared to siNC (Fig. 8A). As shown in Fig. 8B and C, circRNA0071196 silencing significantly reduced the viability and migration of BCa cells $(\mathrm{P}<0.05)$, which was rescued by miR-19b-3p.
CIT is correlated with BCa progression. To determine the relevance of CIT in $\mathrm{BCa}$, we analyzed the in situ levels in tumor and normal bladder tissues, and found that 73 of 80 (91.3\%) BCa tissues expressed this protein. Furthermore, CIT expression was found to be significantly correlated with metastasis $(\mathrm{P}<0.05)$ and histological grade $(\mathrm{P}<0.05)$ but not with age, sex, infiltration and $\mathrm{T}$ stage (Table II). In addition, the intensity of CIT expression increased across grade 1 (least aggressive), 2 (moderately aggressive) and 3 grade (most aggressive and most likely to spread) tumors (Fig. 10A and B), indicating that the CIT level in tumor tissues can demarcate BCa patients into different grades. Since CIT mRNA was upregulated in BCa tissues compared to that noted in the normal tissues (Fig. 5A), to determine its functional relevance in $\mathrm{BCa}$, we knocked down CIT in 5637 cells (Fig. 9A-C). Both circRNA_0071196 and miRNA-19b-3p were down-regulated in sh-CIT cells (Fig. 9D and E), thus underscoring the ceRNA network. Furthermore, CIT knockdown significantly suppressed the proliferative capacity (Fig. 10C), in vitro migration (Fig. 10D) and colony forming capacity (Fig. 10E) of BCa cells, and upregulated p53 and p-ERK1/2, However, the levels of myosin light chain 2 (MLC2), cyclin-dependent kinase 1 (CDK1), murine double minute 2 (MDM2), cyclin D1 (CCND1) and rho-associated, coiled-coil-containing protein kinase 1 (ROCK1) were decreased in sh-CIT group (Fig. 11A-C). 


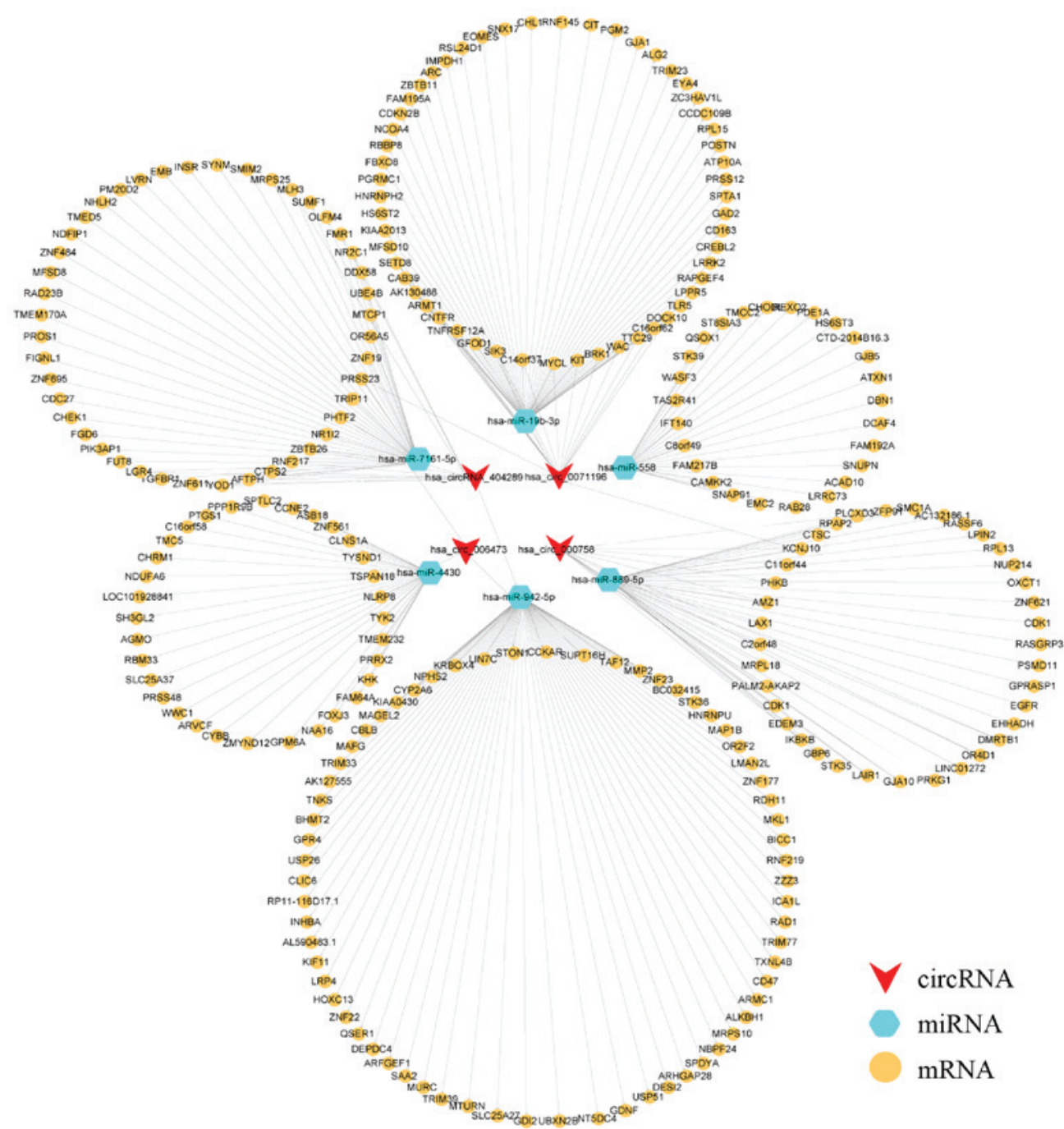

Figure 6. The circRNA/miRNA/mRNA network. Each gene corresponds to a node, and 2 interacting genes based on seed sequence pairings are connected by a solid line. The ' $\mathrm{V}$ ' shapes represent circRNAs, the hexagons represent miRNAs and the circles represent mRNAs. circRNAs, circulating RNAs; miRNAs, microRNAs.
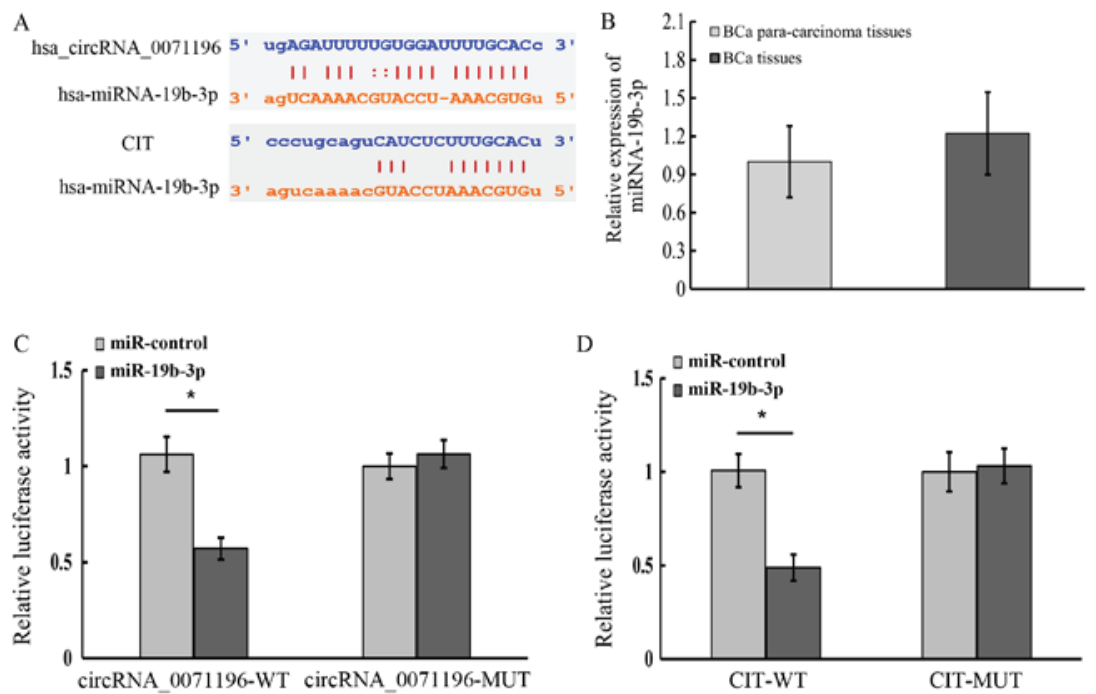

Figure 7. circRNA_0071196 and CIT interact with miR-19b-3p in BCa cells. (A) The sequences of circRNA_0071196/miR-19b-3p and CIT/miR-19b-3p indicating complementary regions. (B) Expression level of miRNA19b-3p exhibits no difference in BCa tissues compared to adjacent normal tissues. (C) Luciferase activity in 5637 cells co-transfected with control luciferase reporter plasmid or the reporter plasmids containing circRNA_0071196 WT 3'-UTR or circRNA_0071196 MUT 3'-UTR, and miR-19b-3p or miR-control vector after 48 h. (D) Luciferase activity in 5637 cells co-transfected with control luciferase reporter plasmid or the reporter plasmid containing CIT WT 3'-UTR or CIT MUT 3'-UTR, and miR-19b-3p or miR-control vector after $48 \mathrm{~h}$. "P<0.05. circRNAs, circulating RNAs; CIT, citron Rho-interacting serine/threonine kinase; WT, wild-type; MUT, mutant; UTR, untranslated region. 

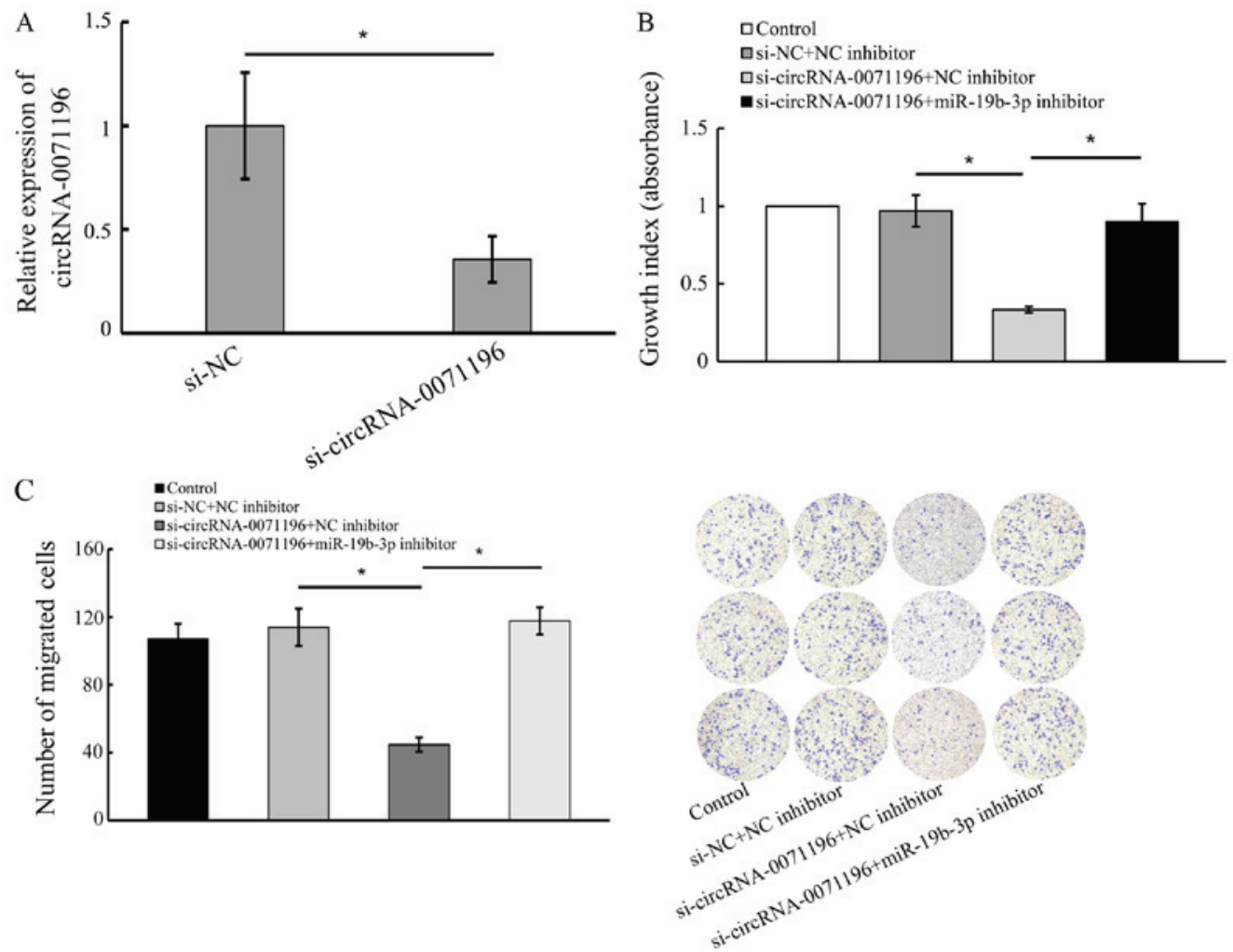

Figure 8. circRNA_0071196 and miR-19b-3p are correlated with BCa progression. (A) The expression levels of circRNA-0071196 following knockdown. Silencing of circular RNA circRNA_0071196 restrained the ability of proliferation (B) and migration (C) compared with the si-NC+NC inhibitor and si-circRNA-0071196+miR-19b-3p inhibitor in 5637 cells ("P<0.05). BCa, bladder cancer.

A
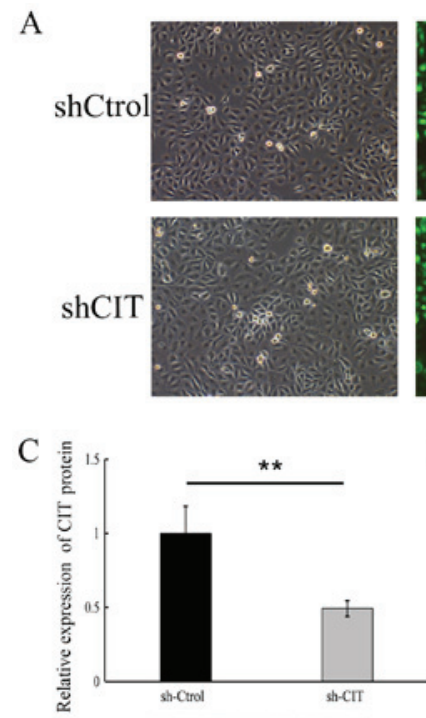

CIT

$\beta$-actin
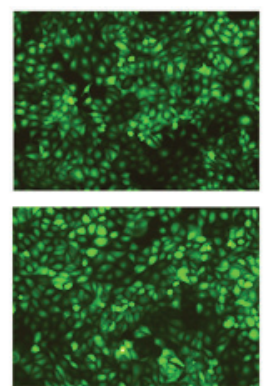

D

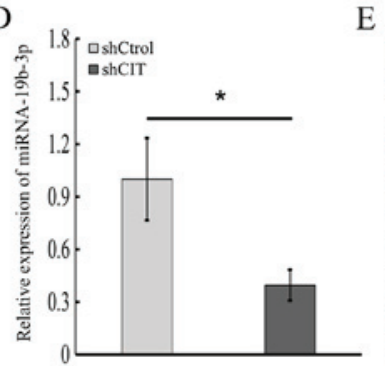

E
B
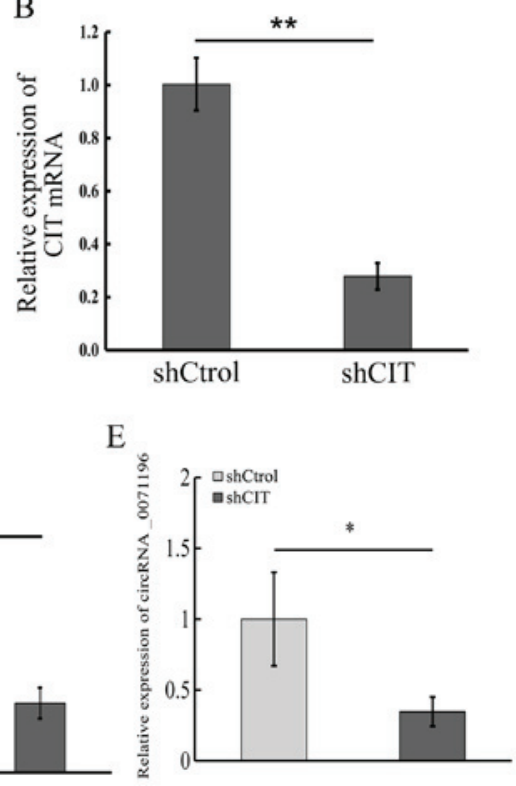

Figure 9. Effect of CIT knockdown in 5637 cells. (A) Representative fluorescence image showing transduced cells. (B and C) The expression levels of CIT mRNA and protein following CIT knockdown. (D and E) The expression levels of miRNA-19b-3p and circRNA_0071196 following CIT knockdown. ${ }^{*} \mathrm{P}<0.05$, ${ }^{* *} \mathrm{P}<0.01$ vs. sh-Ctrol. CIT, citron Rho-interacting serine/threonine kinase.

\section{Discussion}

To elucidate the molecular mechanisms of bladder cancer $(\mathrm{BCa})$ and identify novel diagnostic markers, we explored the mRNA and circRNA expression profiles of matched $\mathrm{BCa}$ and normal bladder tissues. A total of 127 circRNAs and 1,612 mRNAs were differentially expressed in the tumor tissues relative to the healthy tissues, and were functionally annotated primarily 
Table II. CIT expression and clinicopathological characteristics of the bladder cancer cases $(\mathrm{n}=80)$.

\begin{tabular}{|c|c|c|c|c|}
\hline \multirow[b]{2}{*}{ Parameter } & \multirow[b]{2}{*}{ Total (\%) } & \multicolumn{3}{|c|}{ CIT expression } \\
\hline & & Positive $(\mathrm{n}=73) \mathrm{n}(\%)$ & Negative $(n=7)$ n $(\%)$ & P-value \\
\hline Age (years) & & & & 0.676 \\
\hline$>60$ & $54(67.5)$ & $50(92.6)$ & $4(7.4)$ & \\
\hline$\leq 60$ & $26(32.5)$ & $23(88.5)$ & $3(11.5)$ & \\
\hline Sex & & & & 0.415 \\
\hline Male & $55(68.8)$ & $49(89.1)$ & $6(10.9)$ & \\
\hline Female & $25(31.2)$ & $24(96.0)$ & $1(4.0)$ & \\
\hline Metastasis & & & & 0.045 \\
\hline Yes & $43(53.8)$ & $42(97.7)$ & $1(2.3)$ & \\
\hline No & $37(46.2)$ & $31(83.8)$ & $6(16.2)$ & \\
\hline Infiltrative & & & & 0.610 \\
\hline Yes & 15 (18.8) & $13(86.7)$ & $2(13.3)$ & \\
\hline No & $65(81.2)$ & $60(92.3)$ & $5(7.7)$ & \\
\hline Histological grade & & & & 0.011 \\
\hline G1 & $43(53.8)$ & $42(97.7)$ & $1(2.3)$ & \\
\hline G2 & $18(22.5)$ & $17(94.4)$ & $1(5.6)$ & \\
\hline G3 & $19(23.8)$ & $14(73.7)$ & $5(26.3)$ & \\
\hline T stage & & & & 0.668 \\
\hline $\mathrm{T} 1-\mathrm{T} 2$ & $61(76.3)$ & $56(91.8)$ & $5(8.2)$ & \\
\hline T3-T4 & $19(23.8)$ & $17(89.5)$ & $2(10.5)$ & \\
\hline
\end{tabular}

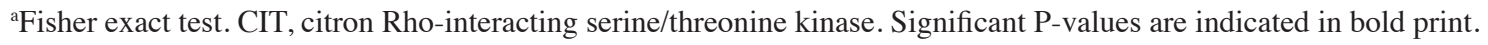

A Control

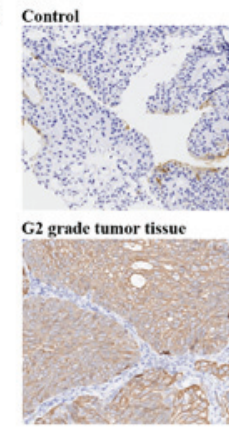

D
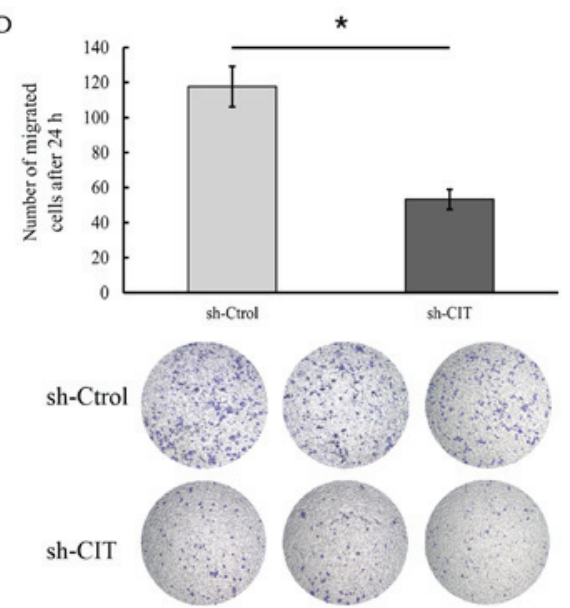

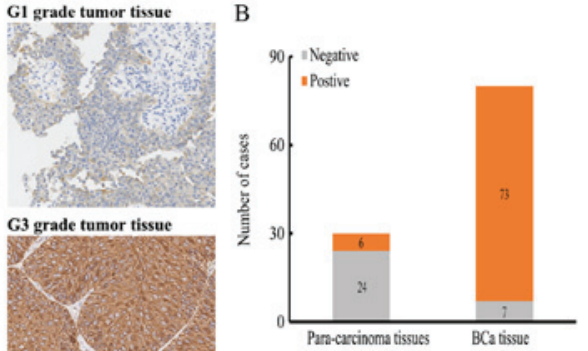

E
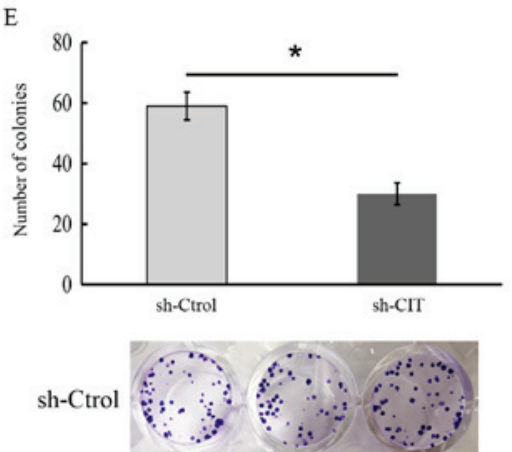

sh-CIT
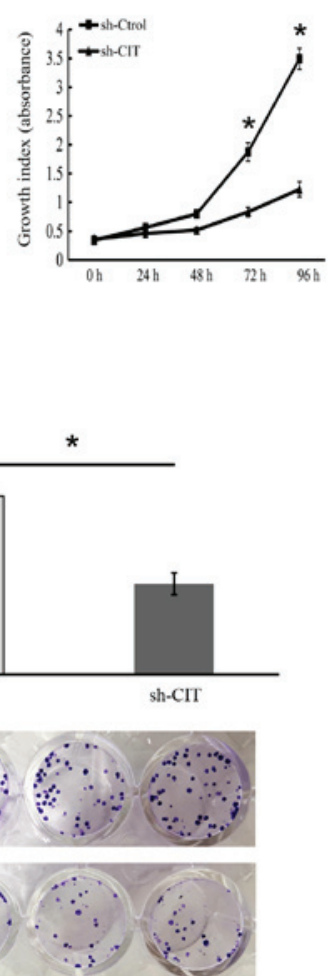

Figure 10. CIT is correlated with BCa progression. (A) Representative immunohistochemistry (IHC) images showing in situ CIT levels in different tumor grades. (B) A number of positive cases of CIT in BCa tissues and adjacent tissues. Results of proliferation (C), migration (D) and colony formation (E) assays in 5637 transfected with sh-CIT or sh-control. ${ }^{*} \mathrm{P}<0.05$ vs. sh-Ctrol. CIT, citron Rho-interacting serine/threonine kinase; BCa, bladder cancer. 

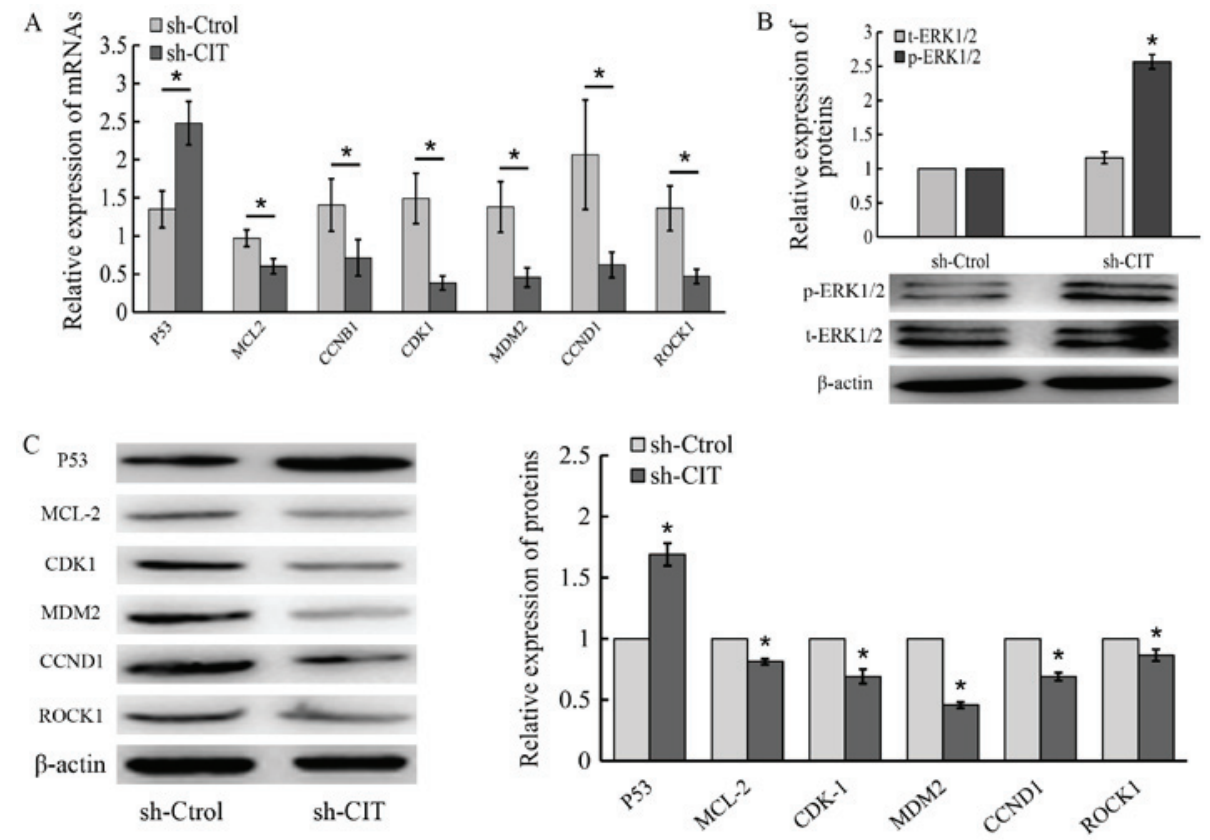

D

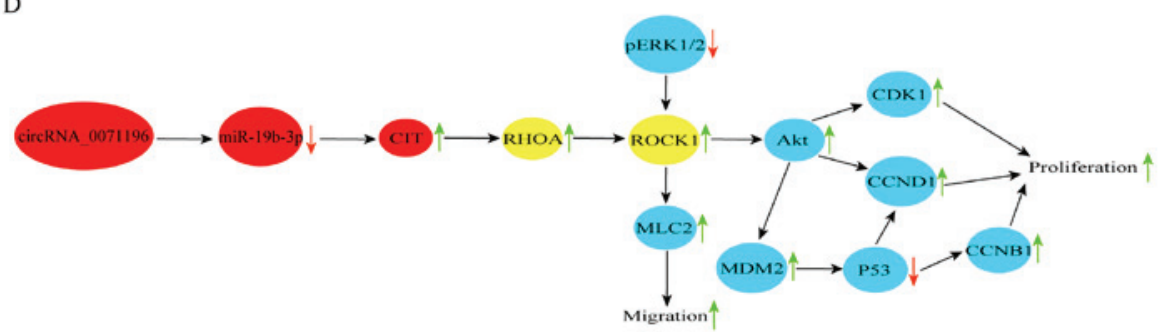

Figure 11. Molecular basis of CIT action in BCa cells. Expression levels of p53, MLC2, CDK1, MDM2, CCND1 and ROCK1 mRNA (A) and protein (B and C) in sh-CIT and sh-Control-transfected 5637 cells. " $\mathrm{P}<0.05$ vs. sh-Control. (D) The schematic diagram of the crosstalk between circRNA_0071196, miR-19b-3p, CIT and ROCK in BCa. CIT, citron Rho-interacting serine/threonine kinase; BCa, bladder cancer; MLC2, myosin light chain 2; CDK1, cyclin-dependent kinase 1; MDM2, murine double minute 2; CCND1, cyclin D1; ROCK2, rho-associated, coiled-coil-containing protein kinase 1; CCNB1, cyclin B1.

to cell metabolism and DNA transcription. In addition, the differentially expressed genes (DEGs) were significantly associated with tumorigenic pathways, which is consistent with the findings of Huang et al (24).

In agreement with previous studies (24-26), the circRNAs MYLK, PC and PTK2 were found to be significantly upregulated in $\mathrm{BCa}$ tissues, and we showed for the first time that circRNA-0071196 is upregulated in BCa versus normal bladder tissues. We next constructed a ceRNA network using the DEcircRNAs, DEGs and the putative miRNAs (27-30), which identified miR-19b-3p as the target of circRNA-10378. It is part of the miR-17-92 cluster that regulates cancer-related pathways $(31,32)$ and vascular remodeling $(22,28,33)$. In addition, miR-19b triggers apoptosis in mouse leukemia cells, and downregulates PTEN in human breast carcinoma $(34,35)$. Niu et al (36) reported a negative correlation between miR-19a/19b and RhoB expression in clear cell renal cell carcinoma specimens and cell lines, indicating that it likely acts as a tumor suppressor. In our study however, we observed no significant difference in the miR-19b-3p levels between BCa and normal bladder tissues. Bioinformatics analysis also predicted citron Rho-interacting serine/threonine kinase (CIT) as the target of miR-19b-3p, and consistent with this, it was aberrantly overexpressed in the $\mathrm{BCa}$ tissues and cell lines. CIT is the downstream effector of Rho family GTPases that are involved in cell cycle regulation (37), and its kinase activity and scaffolding function are vital to cytokinesis $(38,39)$. Previous studies have demonstrated that CIT is significantly upregulated in hepatocellular carcinoma (HCC), and its knockdown inhibited the growth and tumorigenicity of HCC cells (40). Furthermore, the absence of CIT inhibited G1/S transition in colon cancer cells, and the G2/M transition in rat hepatocytes $(23,40)$. At the molecular level, CIT promotes the growth of cancer cells by blocking the p53 pathway (23).

The expression of circRNA-0071196 and miR-19b-3p were decreased by the knockdown of CIT, Currently, the understanding of the degradation mechanism of circRNA is extremely limited. Since circRNAs lack free 5' and 3' ends, endoribonucleolytic cleavage is the only way to degrade circRNAs. Previous studies have demonstrated that N6-methyladenosine (m6A) is associated with degradation of circRNAs through YTHDF2 (m6A reader protein) (41). Yet, the relationship between sh-CIT and m6A is uncertain. Knockdown of CIT inhibited the proliferation, migration and colony forming capacity of 5637 cells, which is similar to the findings in HCC cells (40). Futhermore, CIT knockdown also decreased ROCK1, MLC2, CCNB1, MDM2 and CCND1 levels. P53 and MDM2 inhibit tumor progression and induce regression of established tumors via cell cycle arrest and apoptosis (42). In addition, CIT is an established upstream regulator of RhoA in the late stages of cell division (43), and Sanz-Moreno et al (44) 
indicated that high levels of Rho-ROCK in cancer cells increase actomyosin contractility and promote cell migration. The schematic diagram of the crosstalk between circRNA_0071196, miR-19b-3p, CIT and ROCK in BCa cells is shown in Fig. 11D. Taken together, our findings indicate that circRNA-0071196 upregulates CIT levels in BCa by sponging off miRNA-19b-3p, and the circRNA_0071196/miRNA-19b-3p/CIT axis is a potential therapeutic target in $\mathrm{BCa}$.

\section{Acknowledgements}

The authors thank Ms. Yan Zhang for her technical support.

\section{Funding}

The present study was supported in part by a grant from the Natural Science Foundation of Heilongjiang Province of China (grant nos. ZD201516 and QC2014C112), China Postdoctoral Science Foundation (grant no. 2016M601450), and Heilongjiang Province Postdoctoral Science Foundation (grant no. LBH-Z16139).

\section{Availability of data and materials}

The datasets used during the present study are available from the corresponding author upon reasonable request.

\section{Authors' contributions}

YX conceived and designed the study. ZL, YY, ZY, SX, DL and $\mathrm{BX}$ performed the experiments. $\mathrm{ZL}$ and $\mathrm{YY}$ wrote the paper. YX, ZL and YY reviewed and edited the manuscript. All authors read and approved the manuscript and agree to be accountable for all aspects of the research in ensuring that the accuracy or integrity of any part of the work are appropriately investigated and resolved.

\section{Ethics approval and consent to participate}

The study was approved by the Ethics Committee of The First Affiliated Hospital of Harbin Medical University, and written informed consent was obtained from all patients.

\section{Patient consent for publication}

Not applicable.

\section{Competing interests}

The authors state that they have no competing interests.

\section{References}

1. Bray F, Ferlay J, Soerjomataram I, Siegel RL, Torre LA and Jemal A: Global cancer statistics 2018: GLOBOCAN estimates of incidence and mortality worldwide for 36 cancers in 185 countries. CA Cancer J Clin 68: 394-424, 2018.

2. Spiess PE, Agarwal N, Bangs R, Boorjian SA, Buyyounouski MK, Clark PE, Downs TM, Efstathiou JA, Flaig TW, Friedlander T, et al: Bladder Cancer, Version 5.2017, NCCN Clinical Practice Guidelines in Oncology. J Natl Compr Canc Netw 15: 1240-1267, 2017.
3. Cumberbatch MGK, Jubber I, Black PC, Esperto F, Figueroa JD, Kamat AM, Kiemeney L, Lotan Y, Pang K, Silverman DT, et al: Epidemiology of bladder cancer: A systematic review and contemporary update of risk factors in 2018. Eur Urol 74: 784-795, 2018.

4. Pang C, Guan Y, Li H, Chen W and Zhu G: Urologic cancer in China. Jpn J Clin Oncol 46: 497-501, 2016.

5. Racioppi M, D'Agostino D, Totaro A, Pinto F, Sacco E, D'Addessi A, Marangi F, Palermo G and Bassi PF: Value of current chemotherapy and surgery in advanced and metastatic bladder cancer. Urol Int 88: 249-258, 2012.

6. Dy GW, Gore JL, Forouzanfar MH, Naghavi $M$ and Fitzmaurice C: Global burden of urologic cancers, 1990-2013. Eur Urol 71: 437-446, 2017.

7. Chen Z, Luo Y, Yang W, Ding L, Wang J, Tu J, Geng B, Cui Q and Yang J: Comparison analysis of dysregulated lncrna profile in mouse plasma and liver after hepatic ischemia/reperfusion injury. PLoS One 10: e0133462, 2015.

8. Liz J and Esteller M: lncRNAs and microRNAs with a role in cancer development. Biochim Biophys Acta 1859: 169-176, 2016.

9. Qu S, Yang X, Li X, Wang J, Gao Y, Shang R, Sun W, Dou K and Li H: Circular RNA: A new star of noncoding RNAs. Cancer Lett 365: 141-148, 2015.

10. Li J, Yang J, Zhou P, Le Y, Zhou C, Wang S, Xu D, Lin HK and Gong Z: Circular RNAs in cancer: Novel insights into origins, properties, functions and implications. Am J Cancer Res 5: 472-480, 2015 .

11. Zhao ZJ and Shen J: Circular RNA participates in the carcinogenesis and the malignant behavior of cancer. RNA Biol 14: 514-521, 2017.

12. Guo JU, Agarwal V, Guo H and Bartel DP: Expanded identification and characterization of mammalian circular RNAs. Genome Biol 15: 409, 2014.

13. Salmena L, Poliseno L, Tay Y, Kats L and Pandolfi PP: A ceRNA hypothesis: The Rosetta Stone of a hidden RNA language? Cell 146: 353-358, 2011.

14. Peng H, Lu M and Selaru FM: The genome-wide gene expression profiling to predict competitive endogenous RNA network in hepatocellular cancer. Genom Data 4: 93-95, 2015.

15. Wang $\mathrm{YH}, \mathrm{Yu} \mathrm{XH}$, Luo SS and Han $\mathrm{H}$ : Comprehensive circular RNA profiling reveals that circular RNA100783 is involved in chronic CD28-associated CD8(+)T cell ageing. Immun Ageing 12: 17, 2015.

16. Fabian MR, Sonenberg N and Filipowicz W: Regulation of mRNA translation and stability by microRNAs. Annu Rev Biochem 79: 351-379, 2010.

17. Sun X, Jiao X, Pestell TG, Fan C, Qin S, Mirabelli E, Ren H and Pestell RG: MicroRNAs and cancer stem cells: The sword and the shield. Oncogene 33: 4967-4977, 2014.

18. Liu Y, Li M, Zhang G and Pang Z: MicroRNA-10b overexpression promotes non-small cell lung cancer cell proliferation and invasion. Eur J Med Res 18: 41, 2013.

19. Livak KJ and Schmittgen TD: Analysis of relative gene expression data using real-time quantitative PCR and the 2(- $\Delta \Delta$ C(T)) method. Methods 25: 402-408, 2001.

20. Du H, Shi J, Wang M, An S, Guo X and Wang Z: Analyses of gene expression profiles in the rat dorsal horn of the spinal cord using RNA sequencing in chronic constriction injury rats. J Neuroinflammation 15: 280, 2018. [J].

21. Shannon P, Markiel A, Ozier O, Baliga NS, Wang JT, Ramage D, Amin N, Schwikowski B and Ideker T: Cytoscape: a software environment for integrated models of biomolecular interaction networks. Genome Research 13:2498-2504, 2003.

22. Jin J, Sun Z, Yang F, Tang L, Chen W and Guan X: miR-19b-3p inhibits breast cancer cell proliferation and reverses saracatinib-resistance by regulating PI3K/Akt pathway. Arch Biochem Biophys 645: 54-60, 2018.

23. Wu Z, Zhu X, Xu W, Zhang Y, Chen L, Qiu F, Zhang B, Wu L, Peng $Z$ and Tang H: Up-regulation of CIT promotes the growth of colon cancer cells. Oncotarget 8: 71954-71964, 2017.

24. Huang M,Zhong Z,Lv M,Shu J,Tian Q and Chen J: Comprehensive analysis of differentially expressed profiles of lncRNAs and circRNAs with associated co-expression and ceRNA networks in bladder carcinoma. Oncotarget 7: 47186-47200, 2016.

25. Li Y, Zheng F, Xiao X, Xie F, Tao D, Huang C, Liu D, Wang M, Wang L, Zeng F, et al: CircHIPK3 sponges miR-558 to suppress heparanase expression in bladder cancer cells. EMBO Rep 18: 1646-1659, 2017

26. Yang C, Yuan W, Yang X, Li P, Wang J, Han J, Tao J, Li P, Yang H, Lv Q, et al: Circular RNA circ-ITCH inhibits bladder cancer progression by sponging miR-17/miR-224 and regulating p21, PTEN expression. Mol Cancer 17: 19, 2018. 
27. Xu J, Wang Y, Tan X and Jing H: MicroRNAs in autophagy and their emerging roles in crosstalk with apoptosis. Autophagy 8: 873-882, 2012

28. Lan T, Li C, Yang G, Sun Y, Zhuang L, Ou Y, Li H, Wang G, Kisseleva T, Brenner D, et al: Sphingosine kinase 1 promotes liver fibrosis by preventing miR-19b-3p-mediated inhibition of CCR2. Hepatology 68: 1070-1086, 2018.

29. Selcuklu SD, Donoghue MT, Rehmet K, de Souza Gomes M, Fort A, Kovvuru P, Muniyappa MK, Kerin MJ, Enright AJ and Spillane C: MicroRNA-9 inhibition of cell proliferation and identification of novel miR-9 targets by transcriptome profiling in breast cancer cells. J Biol Chem 287: 29516-29528, 2012.

30. Su Z, Yang Z, Xu Y, Chen Y and Yu Q: MicroRNAs in apoptosis, autophagy and necroptosis. Oncotarget 6: 8474-8490, 2015.

31. Fichtlscherer S, De Rosa S, Fox H, Schwietz T, Fischer A, LiebetrauC, Weber M, Hamm CW, Röxe T, Müller-Ardogan $\mathrm{M}$, et al: Circulating microRNAs in patients with coronary artery disease. Circ Res 107: 677-684, 2010.

32. Olive V, Sabio E, Bennett MJ, De Jong CS, Biton A, McGann JC, Greaney SK, Sodir NM, Zhou AY, Balakrishnan A, et al: A component of the mir-17-92 polycistronic oncomir promotes oncogene-dependent apoptosis. eLife 2: e00822, 2013.

33. Wang WB, Li HP, Yan J, Zhuang F, Bao M, Liu JT, Qi YX and Han Y: CTGF regulates cyclic stretch-induced vascular smooth muscle cell proliferation via microRNA-19b-3p. Exp Cell Res 376: 77-85, 2019.

34. Mavrakis KJ, Wolfe AL, Oricchio E, Palomero T, de Keersmaecker K, McJunkin K, Zuber J, James T, Khan AA, Leslie CS, et al: Genome-wide RNA-mediated interference screen identifies miR-19 targets in Notch-induced T-cell acute lymphoblastic leukaemia. Nat Cell Biol 12: 372-379, 2010.

35. Liang Z, Li Y, Huang K, Wagar N and Shim H: Regulation of miR-19 to breast cancer chemoresistance through targeting PTEN. Pharm Res 28: 3091-3100, 2011.
36. Niu S, Ma X, Zhang Y, Liu YN, Chen X, Gong H, Yao Y, Liu K and Zhang X: MicroRNA-19a and microRNA-19b promote the malignancy of clear cell renal cell carcinoma through targeting the tumor suppressor RhoB. PLoS One 13: e0192790, 2018.

37. Wettschureck N and Offermanns S: Rho/Rho-kinase mediated signaling in physiology and pathophysiology. J Mol Med (Berl) 80: 629-638, 2002.

38. El Amine N, Kechad A, Jananji S and Hickson GR: Opposing actions of septins and Sticky on Anillin promote the transition from contractile to midbody ring. J Cell Biol 203: 487-504, 2013.

39. Harding BN, Moccia A, Drunat S, Soukarieh O, Tubeuf H, Chitty LS, Verloes A, Gressens P, El Ghouzzi V, Joriot S, et al: Mutations in citron kinase cause recessive microlissencephaly with multinucleated neurons. Am J Hum Genet 99: 511-520, 2016.

40. Fu Y, Huang J, Wang KS, Zhang X and Han ZG: RNA interference targeting CITRON can significantly inhibit the proliferation of hepatocellular carcinoma cells. Mol Biol Rep 38: 693-702, 2011.

41. Park OH, Ha H, Lee Y, Boo SH, Kwon DH, Song HK and Kim YK: Endoribonucleolytic Cleavage of m6A-Containing RNAs by RNase P/MRP Complex. Mol Cell 74: 494-507.e8, 2019.

42. Chen J: The cell-cycle arrest and apoptotic functions of p53 in tumor initiation and progression. Cold Spring Harb Perspect Med 6: a026104, 2016.

43. Gai M, Camera P, Dema A, Bianchi F, Berto G, Scarpa E, Germena G and Di Cunto F: Citron kinase controls abscission through RhoA and anillin. Mol Biol Cell 22: 3768-3778, 2011.

44. Sanz-Moreno V, Gaggioli C, Yeo M, Albrengues J, Wallberg F, Viros A, Hooper S, Mitter R, Féral CC, Cook M, et al: ROCK and JAK1 signaling cooperate to control actomyosin contractility in tumor cells and stroma. Cancer Cell 20: 229-245, 2011. International (CC BY-NC-ND 4.0) License. 